\title{
Organic acids composition of Cydonia oblonga Miller leaf
}

\author{
Andreia P. Oliveira ${ }^{a}$, José A. Pereira ${ }^{b}$, Paula B. Andrade ${ }^{c}$, Patrícia Valentão ${ }^{c}$, Rosa M. Seabra ${ }^{c}$, \\ Branca M. Silva ${ }^{\mathrm{a}, \mathrm{c}, *}$ \\ ${ }^{a}$ Faculdade de Ciências da Saúde, Universidade Fernando Pessoa, R. Carlos da Maia, 296, 4200-150 Porto, Portugal \\ b CIMO, Escola Superior Agrária, Instituto Politécnico de Bragança, Campus de Santa Apolónia, Apartado 1172, $5301-855$ Bragança, Portugal \\ ${ }^{\mathrm{c}}$ REQUIMTE, Serviço de Farmacognosia, Faculdade de Farmácia, Universidade do Porto, R. Aníbal Cunha, 4050-047 Porto, Portugal
}

\section{A R T I C L E I N F O}

\section{Article history:}

Received 29 January 2008

Received in revised form 22 February 2008

Accepted 1 April 2008

\section{Keywords:}

Cydonia oblonga Miller

Quince leaves

Organic acids

Variability

\begin{abstract}
A B S T R A C T
Organic acid profiles of 36 Cydonia oblonga Miller leaf samples, from three different geographical origins of northern (Bragança and Carrazeda de Ansiães) and central Portugal (Covilhã), harvested in three collection months (June, August and October of 2006), were determined by HPLC/UV (214 nm). Quince leaves presented a common organic acid profile, composed of six constituents: oxalic, citric, malic, quinic, shikimic and fumaric acids. C. oblonga leaves total organic acid content varied from 1.6 to $25.8 \mathrm{~g} / \mathrm{kg} \mathrm{dry}$ matter (mean value of $10.5 \mathrm{~g} / \mathrm{kg}$ dry matter). Quinic acid was the major compound (72.2\%), followed by citric acid (13.6\%).

Significant differences were found in malic and quinic acids relative abundances and total organic acid contents according to collection time, which indicates a possible use of these compounds as maturity markers.

Between June and August seems to be the best period to harvest quince leaves for preparation of decoctions or infusions, since organic acids total content is higher in this season.
\end{abstract}

(c) 2008 Elsevier Ltd. All rights reserved.

\section{Introduction}

Several studies have proved that significant health risks and benefits are associated with dietary food choice (Wildman, 2001; Fattouch et al., 2007). Mediterranean diets are characterized by abundant intakes of plant foods, such as fruits, vegetables, nuts, seeds and wild plants. Biomolecules in these plants have attracted a great deal of attention, mainly concentrated on their role in preventing diseases, such as cancers and cardio- and cerebrovascular disorders (Guthrie \& Kurowska, 2001). This association is often attributed to the antioxidants present in these food products, such as phenolic compounds (phenolic acids and flavonoids), vitamin E, carotenoids, L-ascorbic acid and other organic acids, which prevent free radical damage (du Toit, Volsteedt \& Apostolides, 2001; Silva, Andrade, Valentão et al., 2004).

Interest in edible plants as sources of natural bioactive compounds prompted our research group to investigate the chemical composition of quince fruit and its derivatives (Andrade, Carvalho, Seabra, \& Ferreira, 1998; Ferreres, Silva, Andrade, Seabra, \& Ferreira, 2003; Silva, Andrade, Ferreres et al., 2002; Silva, Andrade, Gonalves et al., 2004; Silva, Andrade, Martins, Seabra, \& Ferreira, 2006; Silva, Andrade, Mendes, Seabra, \& Ferreira, 2002; Silva et al., 2000;

\footnotetext{
* Corresponding author. Address: Faculdade de Ciências da Saúde, Universidade Fernando Pessoa, R. Carlos da Maia, 296, 4200-150 Porto, Portugal. Tel.: +351 225074630; fax: +351 225508269 .

E-mail address: bsilva@ufp.pt (B.M. Silva).
}

Silva, Andrade, Seabra, \& Ferreira, 2001; Silva, Andrade, Valentão et al., 2004; Silva et al., 2000 \& 2003; Silva, Andrade, Martins et al., 2005; Silva, Andrade, Seabra et al., 2005; Sousa et al., 2007), in terms of phenolics, organic acids and free amino acids (by HPLC-DAD and HPLC-DAD-MS/MS-ESI, HPLC/UV and GC-FID, respectively), as well as their antioxidant potential (Silva, Andrade, Valentão et al., 2004).

Nowadays, quince fruit is recognized as a good, cheap and important dietary source of health-promoting compounds, due to its biologically active constituents which are characterized by their antioxidant, antimicrobial and anti-ulcerative properties (Fattouch et al., 2007; García-Alonso, Pascual-Teresa, Santos-Buelga, \& RivasGonzalo, 2004; Hamauzu, Hisako, Takaroni, Kume, \& Omanyuda, 2005; Hamauzu, Takaroni, Kume, Irie, \& Hiramatsu, 2006; Silva, Valentão, Seabra, \& Andrade, in press; Silva, Andrade, Valentão et al., 2004; Wang, Jia, Zhao, \& e Wang, 2006; Yildirim, 2006).

Cydonia oblonga Miller leaves have been used, after decoction or infusion, in folk medicine for their sedative, antipyretic, anti-diarrheic and antitussive properties and for the treatment of various skin diseases (De Tommasi, De Simone, Pizza et al., 1996; Oliveira et al., 2007). Nevertheless, as far as we know, few phytochemical studies have been undertaken in this matrix. Some $\alpha$ - and $\beta$-ionol and flavonol glycosides have been isolated from quince leaves and identified by De Tommasi, De Simone, Pizza et al. (1996), De Tommasi, Piacente, De Simone, et al. (1996b) and Lutz, Schneider, and Winterhalter (2002). In addition, interest in edible plants 
as sources of natural antioxidants prompted us to study the phenolic composition of $C$. oblonga leaves (Oliveira et al., 2007). The phenolic profile was composed of nine compounds: 3-O-, 4$\mathrm{O}$ - and 5-O-caffeoylquinic acids, 3,5-O-dicaffeoylquinic acid, quercetin-3-O-galactoside, quercetin-3-O-rutinoside, kaempferol-3-Oglucoside, kaempferol-3-O-rutinoside and a kaempferol-3-O-glycoside. This study suggested that leaves from $C$. oblonga can be used as a great and cheap source of bioactive compounds and may have relevance in the prevention of diseases in which free radicals are implicated. Significant differences were found in 3-O-caffeoylquinic and 3,5-O-dicaffeoylquinic acid contents, according to geographical origin and collection month, which indicates a possible use of these compounds as markers of samples with different geographical origins and/or physiological maturities (Oliveira et al., 2007).

Organic acids are primary metabolites, which can be found in great amounts in all plants, especially in fruits. Citric, malic and tartaric acids are commonly found in fruits and berries, while oxalic acid is present in higher amounts in green leaves. As phenolics, the organic acids may also have a protective role against various diseases due to their antioxidant properties (Silva, Andrade, Valentão et al., 2004; Valentão, Andrade, Rangel et al., 2005; Valentão, Lopes, Valente et al., 2005). For instance, ascorbic acid (vitamin C) is the most widely distributed water-soluble antioxidant in fruits and vegetables. Oxalic acid is the simplest dicarboxylic acid and its most striking chemical impact is its strong chelating ability for multivalent cations. Other carboxylic acids, such as tartaric, malic, citric, succinic and hydroxyglutaric, behave as antioxidants because they also have the ability to chelate metals. They are, therefore, classified as "preventive" or synergistic (Seabra et al., 2006).

Recently, the total phenolics content of $C$. oblonga leaves was reported as much higher than that found for pulps, peels and seeds, which may indicate that this part of the tree can be much more interesting in terms of health-promoting constituents (Oliveira et al., 2007). So, in continuation of our investigation on this plant species, the work herein represents a contribution to the knowledge of quince leaves' composition, concerning their organic acid profile. Additionally, we studied the possible influence of geographical origin and collection month, on the organic acids content. In this plant species, this is the first time that organic acid composition has been evaluated during the collection period.

\section{Materials and methods}

\subsection{Samples}

Thirty-six healthy quince leaves samples were collected in four different places in each one of the three geographical origins of northern and central Portugal - Bragança, Carrazeda de Ansiães and Covilhã - at the beginning of June, August and October of 2006 (Table 1). Each sample was dried in a stove (Memmert UL6D - Germany) at $30 \pm 2{ }^{\circ} \mathrm{C}$ for 5 days (in the dark). The mean drying yield was $49.82 \%$.

\subsection{Standards}

The standards were from Sigma (St. Louis, MO, USA) and from Extrasynthése (Genay, France). Methanol and hydrochloric acids were obtained from Merck (Darmstadt, Germany) and sulphuric acid were from Pronalab (Lisboa, Portugal). The water was treated in a Milli-Q water purification system (Millipore, Bedford, MA, USA).

\subsection{Solid-phase extraction (SPE) columns}

The Chromabond C18 SPE columns $(70 \mathrm{ml} / 10,000 \mathrm{mg})$ were purchased from Macherey-Nagel (Duren, Germany).

\subsection{Extraction of organic acids}

Extraction was achieved as previously reported (Silva, Andrade, Mendes et al., 2002; Silva, Andrade, Valentão et al., 2004; Silva, Andrade, Gonçalves et al., 2004; Silva, Andrade, Martins et al., 2005; Silva, Andrade, Seabra et al., 2005 \& 2006), but with some modifications. Briefly, each dried sample (ca. $0.5 \mathrm{~g}$ ) was thoroughly mixed with methanol $(10 \times 25 \mathrm{ml})$, at $40^{\circ} \mathrm{C}$. The methanolic extract was filtered, concentrated to dryness, under reduced pressure $\left(40^{\circ} \mathrm{C}\right)$, and redissolved in acid water ( $\mathrm{pH} 2$ with $\left.\mathrm{HCl}\right)(\mathrm{ca} .25 \mathrm{ml})$. The aqueous solution obtained was passed through a SPE C18 column, previously conditioned with $30 \mathrm{ml}$ of methanol and $70 \mathrm{ml}$ of acid water ( $\mathrm{pH} 2$ with $\mathrm{HCl}$ ). The aqueous extract was evaporated to dryness under reduced pressure $\left(40^{\circ} \mathrm{C}\right)$, redissolved in sulphuric acid $0.01 \mathrm{~N}(2 \mathrm{ml})$ and $20 \mu \mathrm{l}$ were analysed by HPLC.

\subsection{HPLC/UV system}

Separation was achieved as reported previously (Silva, Andrade, Mendes et al., 2002; Silva, Andrade, Valentão et al., 2004; Silva, Andrade, Gonçalves et al., 2004; Silva, Andrade, Martins et al., 2005; Silva, Andrade, Seabra et al., 2005 \& 2006; Valentão, Andrade, Rangel et al., 2005; Valentão, Lopes, Valente et al., 2005,), with an analytical HPLC unit (Gilson), using an ion exclusion column Nucleogel ${ }^{\circledR}$ Ion 300 OA $(300 \times 7.7 \mathrm{~mm})$, in conjunction with a column heating device at $30^{\circ} \mathrm{C}$. Elution was carried out at a solvent flow rate of $0.2 \mathrm{ml} / \mathrm{min}$, isocratically, with $0.01 \mathrm{~N}$ sulphuric acid as the mobile phase. Detection was performed with a Gilson UV detector at $214 \mathrm{~nm}$.

Table 1

Quince leaves samples characterization

\begin{tabular}{|c|c|c|}
\hline Sample identification & Geographical origin & Collection month \\
\hline 1 & Bragança-Pinheiro Manso & June \\
\hline 2 & Bragança-Pinheiro Manso & August \\
\hline 3 & Bragança-Pinheiro Manso & October \\
\hline 4 & Bragança-Quinta & June \\
\hline 5 & Bragança-Quinta & August \\
\hline 6 & Bragança-Quinta & October \\
\hline 7 & Bragança-Tecnologia & June \\
\hline 8 & Bragança-Tecnologia & August \\
\hline 9 & Bragança-Tecnologia & October \\
\hline 10 & Bragança-Vale de Álvaro & June \\
\hline 11 & Bragança-Vale de Álvaro & August \\
\hline 12 & Bragança-Vale de Álvaro & October \\
\hline 13 & Carrazeda de Ansiães-Barrancas & June \\
\hline 14 & Carrazeda de Ansiães-Barrancas & August \\
\hline 15 & Carrazeda de Ansiães-Barrancas & October \\
\hline 16 & Carrazeda de Ansiães-Botelho & June \\
\hline 17 & Carrazeda de Ansiães-Botelho & August \\
\hline 18 & Carrazeda de Ansiães-Botelho & October \\
\hline 19 & Carrazeda de Ansiães-Cortinha & June \\
\hline 20 & Carrazeda de Ansiães-Cortinha & August \\
\hline 21 & Carrazeda de Ansiães-Cortinha & October \\
\hline 22 & Carrazeda de Ansiães-Gorgulão & June \\
\hline 23 & Carrazeda de Ansiães-Gorgulão & August \\
\hline 24 & Carrazeda de Ansiães-Gorgulão & October \\
\hline 25 & Covilhã-Mina & June \\
\hline 26 & Covilhã-Mina & August \\
\hline 27 & Covilhã-Mina & October \\
\hline 28 & Covilhã-Peso & June \\
\hline 29 & Covilhã-Peso & August \\
\hline 30 & Covilhã-Peso & October \\
\hline 31 & Covilhã-Quinta Ortigal & June \\
\hline 32 & Covilhã-Quinta Ortigal & August \\
\hline 33 & Covilhã-Quinta Ortigal & October \\
\hline 34 & Covilhã-Silveira & June \\
\hline 35 & Covilhã-Silveira & August \\
\hline 36 & Covilhã-Silveira & October \\
\hline
\end{tabular}


Organic acids quantification was achieved by the absorbance recorded in the chromatograms relative to external standards.

\subsection{Statistical analysis}

All statistical analyses involving experimental data were performed by using SAS software (9.1 version). The evaluation of statistical significance was determined by ANOVA, followed by the Tukey LSD test. The level of significance was set at $p \leqslant 0.05$.

\section{Results and discussion}

Results from this study indicate that $C$. Oblonga leaf organic acid profile (qualitative and quantitative), is different from that of quince fruit (pulp, peel and seed) and jam (Silva, Andrade, Mendes

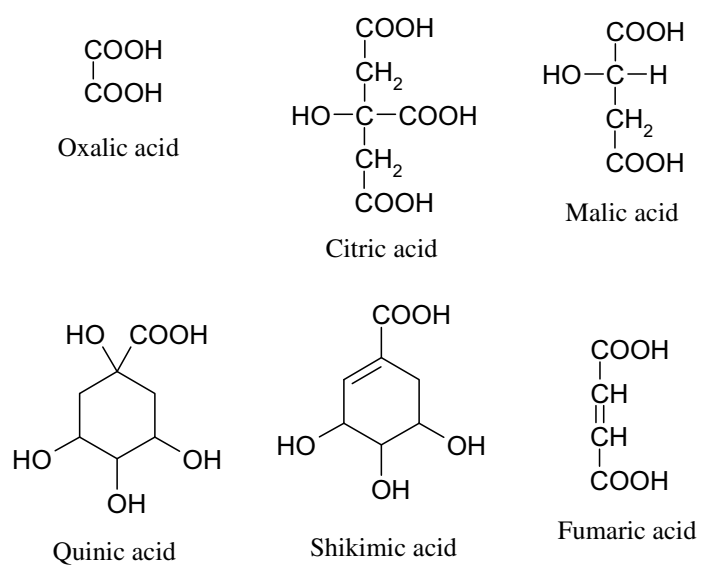

Fig. 1. Organic acids of quince leaves. et al., 2002; Silva, Andrade, Valentão et al., 2004; Silva, Andrade, Gonçalves et al., 2004; Silva, Andrade, Martins et al., 2005; Silva, Andrade, Seabra et al., 2005 \& 2006).

Cydonia oblonga leaf presented a chemical profile composed of six organic acids: oxalic, citric, malic, quinic, shikimic and fumaric acids (Figs. 1 and 2). Quince leaves were rich in quinic acid (72.2\%), had medium values of citric, malic and oxalic acids (mean values of $13.6 \%, 7.6 \%$ and $6.1 \%$, respectively) and very small proportions of shikimic and fumaric acids (<1\%) (Table 2 ).

The total organic acids content varied from 1.6 to $25.8 \mathrm{~g} / \mathrm{kg}$ dry matter (mean value of $10.5 \mathrm{~g} / \mathrm{kg}$ dry matter). Considering the drying yields of quince leaves samples (about 50\%), the total content (between 0.8 and $13 \mathrm{~g} / \mathrm{kg}$ fresh matter; mean value of $5 \mathrm{~g} / \mathrm{kg}$ fresh matter) was lower than those found for pulps (varying from 2 to $17 \mathrm{~g} / \mathrm{kg}$ fresh matter) and peels (between 3 and $16 \mathrm{~g} / \mathrm{kg}$ fresh matter) and higher than that of the seeds (varying from 0.5 to $0.8 \mathrm{~g} / \mathrm{kg}$ fresh matter) (Silva, Andrade, Martins et al., 2005; Silva, Andrade, Seabra et al., 2005).

When compared to quince pulps and peels (Silva, Andrade, Martins et al., 2005, Silva, Andrade, Seabra et al., 2005), leaves presented a characteristic qualitative and quantitative organic acid profile, in which ascorbic acid was absent; the malic plus quinic acids relative content was lower and oxalic, citric, shikimic and fumaric acids relative percentages were higher. Considering quince seeds profile (Silva, Andrade, Seabra et al., 2005), the differences were the presence of oxalic acid, the lower citric and fumaric acid relative contents, the higher malic plus quinic and shikimic acid percentages and absence of ascorbic acid.

It is well known that leaves protect fruits from UV radiation and have an important role in the photosynthesis process, since they are able to convert light energy into chemical energy (glucose and ATP), by scavenging $\mathrm{CO}_{2}$ and producing $\mathrm{O}_{2}$. Later, glucose can be used to produce several metabolites (polysaccharides, amino acids, organic acids, phenolic compounds) according to plant needs. So, it is from leaves that nutrient distribution is carried

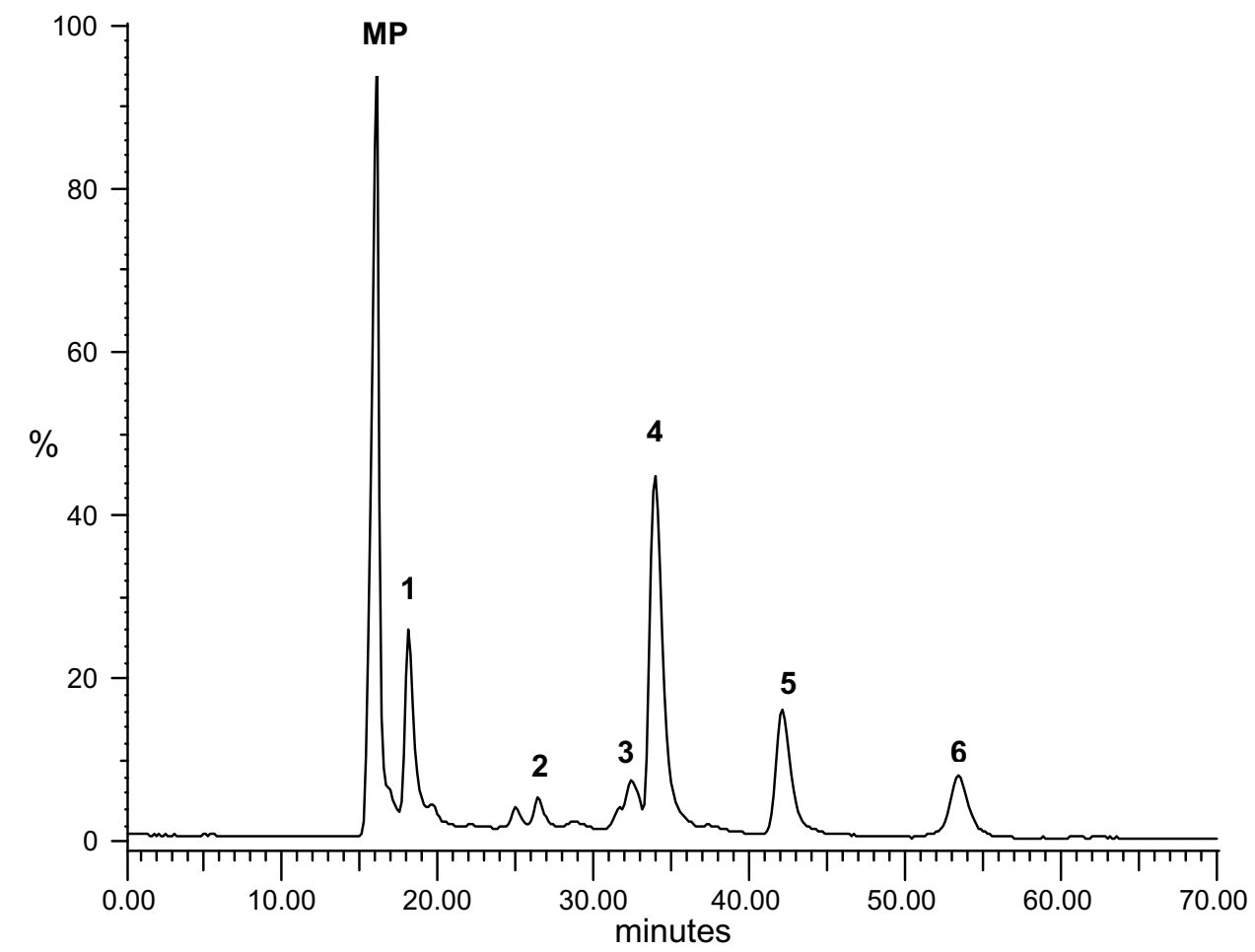

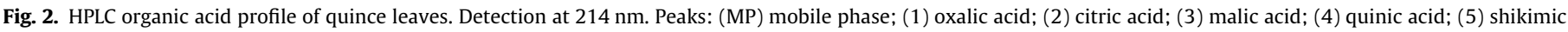
acid; (6) fumaric acid. 
out, to all parts of the tree. In quince leaves, ascorbic acid was not present and the organic acids total content was lower than that found for pulps and peels, but phenolics total content was much higher (Oliveira et al., 2007). Phenolics from quince leaves, namely flavonol derivatives, may have an important role, protecting cells (membranes, chlorophylls and other fragile organelles) from the

Table 2

Organic acids composition of quince leaves samples ${ }^{\mathrm{a}}$

\begin{tabular}{|c|c|c|c|c|c|c|c|}
\hline \multirow[t]{2}{*}{ Sample } & \multicolumn{7}{|c|}{ Organic Acids (\%) } \\
\hline & Oxalic acid & Citric acid & Malic acid & Quinic acid & Shikimic acid & Fumaric acid & $\sum(g / k g)$ \\
\hline 1 & $3.90 \pm 0.07$ & $7.90 \pm 0.42$ & $7.61 \pm 0.04$ & $80.1 \pm 1.51$ & $0.98 \pm 0.01$ & $0.11 \pm 0.01$ & 16.9 \\
\hline 2 & $3.75 \pm 0.04$ & $8.26 \pm 0.10$ & $6.88 \pm 0.06$ & $80.8 \pm 0.62$ & $0.35 \pm 0.01$ & $0.01 \pm 0.00$ & 22.1 \\
\hline 3 & $5.14 \pm 0.23$ & $39.2 \pm 0.27$ & $4.85 \pm 0.03$ & $50.3 \pm 0.38$ & $0.52 \pm 0.01$ & nd & 4.93 \\
\hline 4 & $2.74 \pm 0.03$ & $4.69 \pm 0.19$ & $6.21 \pm 0.03$ & $86.0 \pm 0.67$ & $0.24 \pm 0.00$ & $0.10 \pm 0.01$ & 16.6 \\
\hline 5 & $12.0 \pm 0.56$ & $4.28 \pm 0.04$ & $7.59 \pm 0.02$ & $75.8 \pm 0.37$ & $0.29 \pm 0.01$ & $0.08 \pm 0.01$ & 15.4 \\
\hline 6 & $10.5 \pm 0.14$ & $6.36 \pm 0.24$ & $12.5 \pm 0.52$ & $70.3 \pm 0.26$ & $0.21 \pm 0.01$ & $0.08 \pm 0.01$ & 6.85 \\
\hline 7 & $2.83 \pm 0.28$ & $4.29 \pm 0.08$ & $8.55 \pm 0.01$ & $83.4 \pm 0.08$ & $0.23 \pm 0.01$ & $0.07 \pm 0.01$ & 16.2 \\
\hline 8 & $4.09 \pm 0.01$ & $17.8 \pm 0.46$ & $14.5 \pm 0.05$ & $63.3 \pm 2.00$ & $0.28 \pm 0.01$ & $0.06 \pm 0.01$ & 17.9 \\
\hline 9 & $5.45 \pm 0.07$ & $12.9 \pm 0.09$ & $19.1 \pm 0.03$ & $62.0 \pm 0.62$ & $0.52 \pm 0.02$ & nd & 2.52 \\
\hline 10 & $1.86 \pm 0.10$ & $31.0 \pm 0.45$ & $2.58 \pm 0.17$ & $64.2 \pm 0.28$ & $0.35 \pm 0.01$ & nd & 10.4 \\
\hline 11 & $10.5 \pm 0.27$ & $11.9 \pm 0.37$ & $11.0 \pm 0.32$ & $66.5 \pm 1.23$ & $0.04 \pm 0.01$ & $0.08 \pm 0.01$ & 14.0 \\
\hline 12 & $11.4 \pm 0.56$ & nd & $14.8 \pm 0.46$ & $72.90 \pm 0.19$ & $0.95 \pm 0.03$ & nd & 1.64 \\
\hline 13 & $1.05 \pm 0.01$ & $1.77 \pm 0.13$ & $6.44 \pm 0.07$ & $90.4 \pm 0.73$ & $0.19 \pm 0.00$ & $0.11 \pm 0.00$ & 13.8 \\
\hline 14 & $2.58 \pm 0.03$ & $6.64 \pm 0.08$ & $8.28 \pm 0.09$ & $81.8 \pm 1.45$ & $0.50 \pm 0.01$ & $0.19 \pm 0.01$ & 12.9 \\
\hline 15 & $4.59 \pm 0.05$ & $20.7 \pm 1.18$ & $11.2 \pm 0.05$ & $61.0 \pm 2.62$ & $2.40 \pm 0.12$ & $0.03 \pm 0.01$ & 1.90 \\
\hline 16 & $9.41 \pm 0.47$ & $4.29 \pm 0.24$ & $5.70 \pm 0.24$ & $80.4 \pm 0.03$ & $0.23 \pm 0.01$ & nd & 14.6 \\
\hline 17 & $18.2 \pm 0.63$ & $4.99 \pm 0.14$ & $7.66 \pm 0.03$ & $68.8 \pm 0.26$ & $0.29 \pm 0.01$ & $0.08 \pm 0.01$ & 11.3 \\
\hline 18 & $4.63 \pm 0.24$ & $12.2 \pm 1.15$ & $10.9 \pm 0.10$ & $70.9 \pm 0.97$ & $1.36 \pm 0.01$ & $0.02 \pm 0.01$ & 3.05 \\
\hline 19 & $0.46 \pm 0.01$ & $26.5 \pm 0.02$ & $3.19 \pm 0.08$ & $69.4 \pm 0.11$ & $0.42 \pm 0.01$ & nd & 4.35 \\
\hline 20 & $7.83 \pm 0.10$ & $7.94 \pm 0.31$ & $9.45 \pm 0.23$ & $74.2 \pm 2.85$ & $0.44 \pm 0.02$ & $0.14 \pm 0.01$ & 16.8 \\
\hline 21 & $5.64 \pm 0.11$ & $27.2 \pm 0.48$ & $12.6 \pm 0.38$ & $54.0 \pm 1.51$ & $0.57 \pm 0.01$ & nd & 2.45 \\
\hline 22 & $20.8 \pm 0.04$ & $26.2 \pm 0.09$ & $2.97 \pm 0.01$ & $48.9 \pm 0.39$ & $1.14 \pm 0.01$ & nd & 9.88 \\
\hline 23 & $3.97 \pm 0.02$ & $16.5 \pm 0.13$ & $8.38 \pm 0.09$ & $70.6 \pm 0.89$ & $0.55 \pm 0.01$ & nd & 3.28 \\
\hline 24 & $8.84 \pm 0.16$ & $15.1 \pm 0.41$ & $17.2 \pm 0.01$ & $58.2 \pm 0.29$ & $0.58 \pm 0.02$ & $0.02 \pm 0.01$ & 2.06 \\
\hline 25 & $2.53 \pm 0.07$ & $5.20 \pm 0.03$ & $0.93 \pm 0.04$ & $91.0 \pm 1.91$ & $0.38 \pm 0.01$ & nd & 12.7 \\
\hline 26 & $5.30 \pm 0.04$ & $15.6 \pm 0.01$ & $5.70 \pm 0.06$ & $72.5 \pm 0.29$ & $0.90 \pm 0.01$ & nd & 6.53 \\
\hline 27 & $14.0 \pm 0.20$ & nd & $2.17 \pm 0.02$ & $83.8 \pm 1.72$ & $0.04 \pm 0.01$ & nd & 1.88 \\
\hline 28 & $9.82 \pm 0.02$ & $7.61 \pm 0.45$ & $3.42 \pm 0.03$ & $78.8 \pm 0.04$ & $0.25 \pm 0.01$ & $0.01 \pm 0.01$ & 25.8 \\
\hline 29 & $3.18 \pm 0.01$ & $29.7 \pm 1.26$ & $2.63 \pm 0.10$ & $64.2 \pm 2.41$ & $0.37 \pm 0.02$ & nd & 9.72 \\
\hline 30 & $0.60 \pm 0.04$ & $9.30 \pm 0.05$ & $3.78 \pm 0.08$ & $85.9 \pm 1.79$ & $0.38 \pm 0.01$ & $0.07 \pm 0.00$ & 6.32 \\
\hline 31 & $1.07 \pm 0.03$ & $12.1 \pm 0.07$ & $6.95 \pm 0.14$ & $79.5 \pm 0.12$ & $0.37 \pm 0.01$ & $0.11 \pm 0.01$ & 18.9 \\
\hline 32 & $3.04 \pm 0.30$ & $18.2 \pm 0.15$ & $7.62 \pm 0.72$ & $70.7 \pm 4.42$ & $0.47 \pm 0.01$ & nd & 7.22 \\
\hline 33 & $8.28 \pm 0.14$ & $4.92 \pm 0.11$ & $7.62 \pm 0.15$ & $79.2 \pm 1.92$ & nd & nd & 1.85 \\
\hline 34 & $3.93 \pm 0.07$ & $3.15 \pm 0.01$ & $3.20 \pm 0.17$ & $89.3 \pm 0.86$ & $0.37 \pm 0.01$ & $0.06 \pm 0.01$ & 22.5 \\
\hline 35 & $3.12 \pm 0.02$ & $32.0 \pm 0.39$ & $2.22 \pm 0.04$ & $62.2 \pm 0.40$ & $0.38 \pm 0.03$ & nd & 12.8 \\
\hline 36 & $3.13 \pm 0.13$ & $32.8 \pm 0.01$ & $6.83 \pm 0.13$ & $56.6 \pm 1.82$ & $0.54 \pm 0.01$ & $0.09 \pm 0.01$ & 8.89 \\
\hline Mean & 6.12 & 13.6 & 7.59 & 72.2 & 0.50 & 0.04 & 10.5 \\
\hline Max & 20.8 & 39.2 & 19.1 & 91.0 & 2.40 & 0.19 & 25.8 \\
\hline Min & 0.46 & nd & 0.93 & 48.9 & nd & nd & 1.64 \\
\hline SD & 4.81 & 10.66 & 4.40 & 11.3 & 0.44 & 0.05 & 6.77 \\
\hline
\end{tabular}

${ }^{\text {a }}$ Values (as percentages) are expressed as mean \pm standard deviation of three assays for each sample. Abreviations: nd, not detected; SD, standard deviation; $\sum$, sum of the determined organic acids.

Table 3

Organic acids composition of quince leaves, according to collection month (means \pm SD)

\begin{tabular}{|c|c|c|c|c|c|c|c|}
\hline \multirow[t]{2}{*}{ Month } & \multicolumn{7}{|c|}{ Organic acids (\%) } \\
\hline & Oxalic acid & Citric Acid & Malic acid & Quinic acid & Shikimic acid & Fumaric acid & $\sum(g / k g)$ \\
\hline \multicolumn{8}{|l|}{ Bragança } \\
\hline June & $2.83 \pm 0.84 a$ & $12.0 \pm 12.79 \mathrm{a}$ & $6.24 \pm 2.62 \mathrm{a}$ & $78.4 \pm 9.78 a$ & $0.30 \pm 0.08 \mathrm{a}$ & $0.07 \pm 0.05 a$ & $15.0 \pm 3.07 \mathrm{a}$ \\
\hline August & $7.59 \pm 4.28 \mathrm{a}$ & $10.6 \pm 5.76 \mathrm{a}$ & $9.99 \pm 3.49 a$ & $71.6 \pm 8.10 \mathrm{a}$ & $0.24 \pm 0.14 \mathrm{a}$ & $0.06 \pm 0.03 a$ & $17.3 \pm 3.56 \mathrm{a}$ \\
\hline October & $8.13 \pm 3.29 a$ & $14.6 \pm 17.2 \mathrm{a}$ & $12.8 \pm 5.96 \mathrm{a}$ & $63.9 \pm 10.2 \mathrm{a}$ & $0.55 \pm 0.30 \mathrm{a}$ & $0.02 \pm 0.04 a$ & $3.99 \pm 2.36 b$ \\
\hline \multicolumn{8}{|c|}{ Carrazeda de Ansiães } \\
\hline June & $7.93 \pm 9.50 \mathrm{a}$ & $14.7 \pm 13.5 \mathrm{a}$ & $4.58 \pm 1.75 b$ & $72.3 \pm 17.81 \mathrm{a}$ & $0.50 \pm 0.44 \mathrm{a}$ & $0.03 \pm 0.06 a$ & $10.7 \pm 4.67 \mathrm{a}, \mathrm{b}$ \\
\hline August & $8.14 \pm 7.06 \mathrm{a}$ & $9.01 \pm 5.11 \mathrm{a}$ & $8.44 \pm 2.57 b$ & $73.9 \pm 5.76 \mathrm{a}$ & $0.45 \pm 0.11 \mathrm{a}$ & $0.10 \pm 0.08 a$ & $11.1 \pm 5.67 \mathrm{a}$ \\
\hline October & $5.93 \pm 2.00 \mathrm{a}$ & $18.8 \pm 6.61 \mathrm{a}$ & $13.0 \pm 2.92 \mathrm{a}$ & $61.0 \pm 7.16 \mathrm{a}$ & $1.23 \pm 0.86 a$ & $0.02 \pm 0.01 \mathrm{a}$ & $2.37 \pm 0.51 \mathrm{~b}$ \\
\hline \multicolumn{8}{|l|}{ Covilhã } \\
\hline June & $4.34 \pm 3.84 \mathrm{a}$ & $7.00 \pm 3.83 a$ & $3.63 \pm 2.49 \mathrm{a}$ & $84.7 \pm 6.36 \mathrm{a}$ & $0.34 \pm 0.06 \mathrm{a}$ & $0.05 \pm 0.05 a$ & $20.0 \pm 5.64 a$ \\
\hline August & $3.66 \pm 1.09 \mathrm{a}$ & $23.9 \pm 8.19 a$ & $4.54 \pm 2.57 \mathrm{a}$ & $67.4 \pm 4.98 \mathrm{a}$ & $0.53 \pm 0.25 \mathrm{a}$ & $0.00 \pm 0.00 \mathrm{a}$ & $9.07 \pm 2.84 \mathrm{~b}$ \\
\hline October & $6.51 \pm 5.94 \mathrm{a}$ & $11.8 \pm 11.5 \mathrm{a}$ & $5.10 \pm 2.56 \mathrm{a}$ & $76.4 \pm 13.4 \mathrm{a}$ & $0.24 \pm 0.26 \mathrm{a}$ & $0.04 \pm 0.05 a$ & $4.74 \pm 3.48 \mathrm{~b}$ \\
\hline \multicolumn{8}{|c|}{ All sites combined } \\
\hline June & $5.03 \pm 5.82 \mathrm{a}$ & $11.2 \pm 10.5 \mathrm{a}$ & $4.81 \pm 2.38 b$ & $78.4 \pm 12.3 a$ & $0.38 \pm 0.25 a$ & $0.05 \pm 0.05 a$ & $15.2 \pm 5.75 a$ \\
\hline August & $6.46 \pm 4.82 \mathrm{a}$ & $14.5 \pm 9.11 \mathrm{a}$ & $7.66 \pm 3.32 \mathrm{a}, \mathrm{b}$ & $70.9 \pm 6.44 \mathrm{a}, \mathrm{b}$ & $0.41 \pm 0.20 \mathrm{a}$ & $0.05 \pm 0.06 a$ & $12.5 \pm 5.29 \mathrm{a}$ \\
\hline October & $6.85 \pm 3.82 \mathrm{a}$ & $15.1 \pm 12.6 \mathrm{a}$ & $10.3 \pm 5.34 a$ & $67.1 \pm 11.8 b$ & $0.67 \pm 0.66 \mathrm{a}$ & $0.03 \pm 0.03 a$ & $3.70 \pm 2.44 \mathrm{~b}$ \\
\hline
\end{tabular}

In the same column, means with different letters are significantly different $(p \leqslant 0.05)$. 
damage caused by UV radiation. Probably, leaves produce these secondary compounds because they are more efficient antioxidants than are organic acids. This is in agreement with previous studies performed by our research group, where we have reported quince fruit (pulp, peel and seed) and jam methanolic extract scavenging effects on 1,1-diphenyl-2-picrylhydrazyl (DPPH) (Silva,
Andrade, Valentão et al., 2004). Methanolic extracts were fractionated into phenolic and organic acid fractions. The results indicated that $\mathrm{IC}_{50}$ of the total methanolic extracts was only correlated with the caffeoylquinic acid total contents and the phenolic fraction always exhibited a stronger antioxidant activity than did the whole methanolic extract.
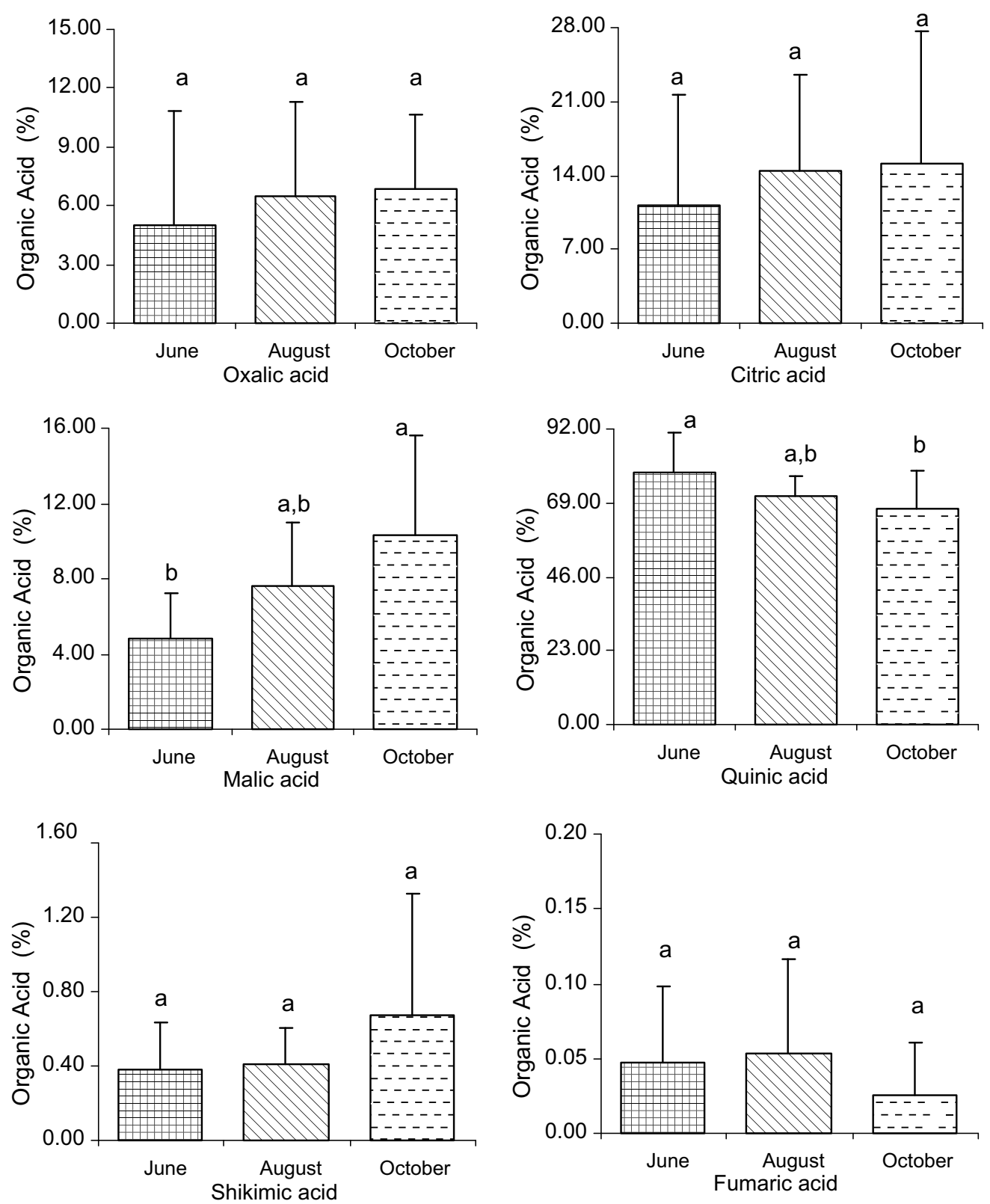

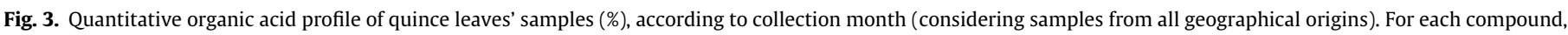
means with different letters are significantly different $(p \leqslant 0.05)$.

Table 4

Organic acids composition of quince leaves, according to geographical origin (means \pm SD)

\begin{tabular}{|c|c|c|c|c|c|c|c|}
\hline \multirow[t]{2}{*}{ Geographical origin } & \multicolumn{7}{|c|}{ Organic acids (\%) } \\
\hline & Oxalic acid & Citric acid & Malic acid & Quinic acid & Shikimic acid & Fumaric acid & $\sum(g / k g)$ \\
\hline Bragança & $6.18 \pm 3.78 a$ & $12.4 \pm 11.73 \mathrm{a}$ & $9.68 \pm 4.77 \mathrm{a}$ & $71.3 \pm 10.5 \mathrm{a}$ & $0.36 \pm 0.23 a$ & $0.05 \pm 0.04 \mathrm{a}$ & $12.1 \pm 6.68 \mathrm{a}$ \\
\hline Carrazeda de Ansiães & $7.33 \pm 6.36 a$ & $14.2 \pm 9.31 \mathrm{a}$ & $8.67 \pm 4.03 \mathrm{a}$ & $69.1 \pm 12.1 \mathrm{a}$ & $0.72 \pm 0.63 \mathrm{a}$ & $0.05 \pm 0.07 \mathrm{a}$ & $8.02 \pm 5.68 \mathrm{a}$ \\
\hline Covilhã & $4.84 \pm 3.93 a$ & $14.2 \pm 11.61 \mathrm{a}$ & $4.42 \pm 2.38 b$ & $76.1 \pm 11.0 \mathrm{a}$ & $0.37 \pm 0.23 a$ & $0.03 \pm 0.04 \mathrm{a}$ & $11.3 \pm 7.68 \mathrm{a}$ \\
\hline
\end{tabular}

In the same column, means with different letters are significantly different $(p \leqslant 0.05)$. 
Significant differences $(p \leqslant 0.05)$ were found among samples harvested in the three different months (concerning malic and quinic acid percentages and total organic acid contents). Samples collected in October presented a lower organic acids total content, considering each geographical origin or all of them at the same time (Table 3; Fig. 3). Generally, the amount of these acids decreased during harvesting time, which can be related to the high temperature verified during summer time, that implies physiological adaptations of plants. Malic and quinic acid contents also varied according to the collection date $(p \leqslant 0.05)$. Leaves' composition, in terms of these two acids, was inverse. Samples which presented high malic acid contents exhibited low quinic acid abundance and vice-versa.

Malic acid content of samples collected in Covilhã (Central Portugal) was significantly lower than those from Bragança and Carrazeda de Ansiães (northern Portugal) samples $(p \leqslant 0.05$ ) (Table 4; Fig. 4).
As far as we know, few chemical studies concerning leaves' organic acid profiles have been developed. Before kombucha tea fermentation (green and black tea leaves), the main organic acid is Dglucuronic acid. Nevertheless, acetic, lactic and citric acids are also found after fermentation and their contents are significant changed during fermentation time (Jayabalan, Marimuthu, \& Swaminathan, 2007). Seven organic acids - aconitic, citric, ascorbic, malic, quinic, shikimic and fumaric acids - were identified and quantified in Brassica oleracea L. var. costata DC internal leaves and citric acid was the main compound (Ferreres et al., 2006) while, in B. oleracea var. costata external leaves, only citric, ascorbic, malic, shikimic and fumaric acids were found, malic acid being the major one (Vrchovská et al., 2006). Rumex induratus leaves were characterized by great oxalic acid concentrations (Ferreres et al., 2006). So, this study suggests that quince leaf has a qualitative and quantitative organic acid profile very distinct from those of other species. As
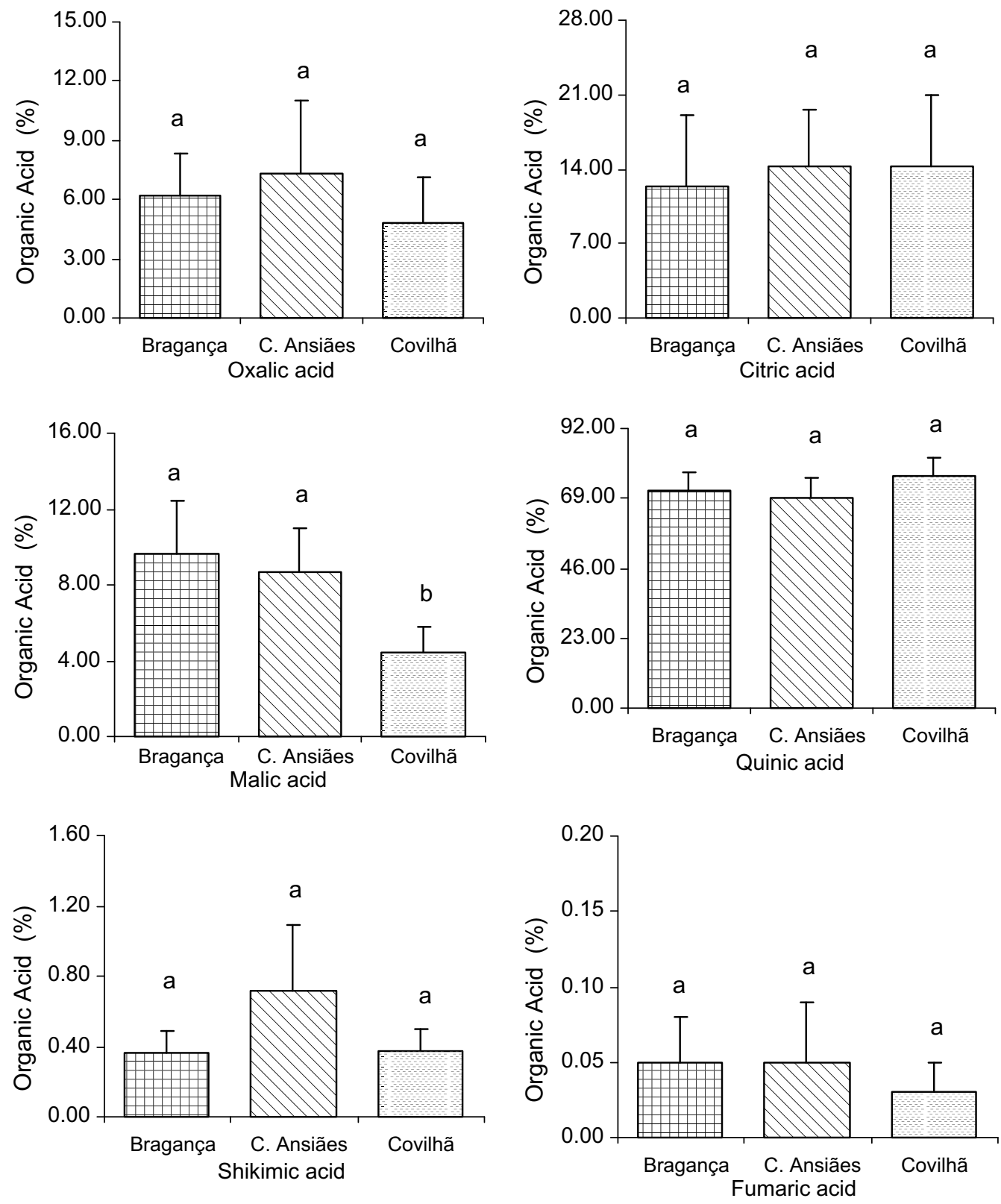

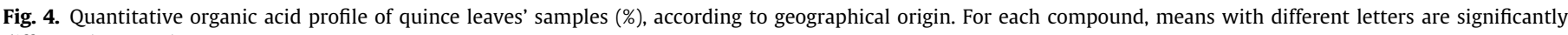
different $(p \leqslant 0.05)$ 
far as we know, nothing about organic acid composition of leaves from trees has been reported.

\section{Conclusion}

Significant differences were observed in malic and quinic acid contents of $C$. oblonga leaves, according to harvesting month, suggesting a possible use of these organic acids as maturity markers.

Between June and August seems to be the best time to collect quince leaves for preparation of decoctions or infusions, since organic acid total contents were higher in this period. Probably, this difference was due to edapho-climatic factors, mainly solar exposure.

Considering quince leaves' composition, in terms of organic acids and phenolic compounds, we intend to progress our studies, in order to evaluate their antioxidant and antimicrobial potential and bioactive compounds in comparison to quince fruit.

\section{References}

Andrade, P. B., Carvalho, A. R. F., Seabra, R. M., \& Ferreira, M. A. (1998). A previous study of phenolic profiles of quince, pear, and apple purees by HPLC diode array detection for the evaluation of quince puree genuineness. Journal of Agricultural and Food Chemistry, 46, 968-972.

De Tommasi, N., De Simone, F., Pizza, C., \& Mahmood, N. (1996). New tetracyclic sesterterpenes from Cydonia vulgaris. Journal of Natural Products, 59, 267-270.

De Tommasi, N., Piacente, S., De Simone, F., \& Pizza, C. (tul=0?>De Tommasi, Piacente, De Simone et al. 1996). Constituents of Cydonia vulgaris: isolation and structure elucidation of four new flavonol glycosides and nine new (ionol-derived glycosides. Journal of Agricultural and Food Chemistry, 44, 1676-1681.

du Toit, R., Volsteedt, Y., \& Apostolides, Z. (2001). Comparison of the antioxidant content of fruits, vegetables and teas measured as vitamin $C$ equivalents. Toxicology, 166, 63-69.

Fattouch, S., Caboni, P., Coroneo, V., Tuberoso, C. I. G., Angioni, A., Dessi, S., et al. (2007). Antimicrobial activity of tunisian quince (Cydonia oblonga Miller) pulp and peel polyphenolic extracts. Journal of Agricultural and Food Chemistry, 55, 963-969.

Ferreres, F., Ribeiro, V., Izquierdo, A. G., Rodrigues, M. A., Seabra, R. M., Andrade, P. B., et al. (2006). Rumex induratus leaves: interesting dietary source of potential bioactive compounds. Journal of Agricultural and Food Chemistry, 54, 5782-5789.

Ferreres, F., Silva, B. M., Andrade, P. B., Seabra, R. M., \& Ferreira, M. A. (2003) Approach to the study of C-glycosyl flavones by ion trap HPLC-PAD-ESI/MS/MS: application to seeds of quince (Cydonia oblonga). Phytochemical Analysis, 14, 352-359.

Ferreres, F., Sousa, C., Vrchovská, V., Valentão, P., Pereira, J. A., Seabra, R. M., et al. (2006). Chemical composition and antioxidant activity of tronchuda cabbage internal leaves. European Food Research and Technology, 222, 88-98.

García-Alonso, M., Pascual-Teresa, S., Santos-Buelga, C., \& Rivas-Gonzalo, J. C. (2004). Evaluation of the antioxidant properties of fruits. Food Chemistry, 84, 13-18.

Guthrie, N., \& Kurowska, E. M. N. (2001). Anticancer and cholesterol-lowering activities of citrus flavonoids. In Handbook of nutraceuticals and functional foods. Florida, Boca Raton: CRC Press.

Hamauzu, Y., Hisako, Y., Takaroni, I., Kume, C., \& Omanyuda, M. (2005). Phenolic profile, antioxidant property, and anti-influenza viral activity of chinese quince (Pseudocydonia sinensis Schneid), quince (Cydonia oblonga Mill.) fruits. Journal of Agricultural and Food Chemistry, 53, 928-934.

Hamauzu, Y., Takaroni, I., Kume, C., Irie, M., \& Hiramatsu, K. (2006). Antioxidant and antiulcerative properties of phenolics from chinese quince, quince, and apple fruits. Journal of Agricultural and Food Chemistry, 54, 765-772.

Jayabalan, R., Marimuthu, S., \& Swaminathan, K. (2007). Changes in content of organic acids and tea polyphenols during kombucha tea fermetation. Food Chemistry, 102, 392-398.
Lutz, A., Schneider, M., \& Winterhalter, P. (2002). Isolation of two new ionone glucosides from quince (Cydonia oblonga Mill) leaves.. Natural Product Letters, $16,119-122$.

Oliveira, A. P., Pereira, J. A., Andrade, P. B., Valentão, P., Seabra, R. M., \& Silva, B. M. (2007). Phenolic profile of Cydonia oblonga Miller leaves. Journal of Agricultural and Food Chemistry, 55, 7926-7930.

Seabra, R. M., Andrade, P. B., Valentão, P., Fernandes, E., Carvalho, F., \& Bastos, M. L. (2006). Anti-oxidant compounds extracted from several plant materials. In Biomaterials from aquatic and terrestrial organisms. New Hampshire: Science Publishers - Enfield (NH) Jersey Plymouth.

Silva, B. M., Andrade, P. B., Ferreres, F., Domingues, A. L., Seabra, R. M., \& Ferreira, M. A. (2002). Phenolic profile of quince fruit (Cydonia oblonga Miller) (pulp and peel). Journal of Agricultural and Food Chemistry, 50, 4615-4618.

Silva, B. M., Andrade, P. B., Gonçalves, A. C., Seabra, R. M., Oliveira, M. B., \& Ferreira, M. A. (2004). Influence of jam processing upon the contents of phenolics, organic acids and free amino acids in quince fruit (Cydonia oblonga Miller). European Food Research and Technology, 218, 385-389.

Silva, B. M., Andrade, P. B., Martins, R. C., Seabra, R. M., \& Ferreira, M. A. (2006). Principal component analysis as tool of characterization of quince (Cydonia oblonga Miller) jam. Food Chemistry, 94, 504-512.

Silva, B. M., Andrade, P. B., Martins, R. C., Valentão, P., Ferreres, F., Seabra, R. M., et al. (2005). Quince (Cydonia oblonga Miller) fruit characterization using principal component analysis. Journal of Agricultural and Food Chemistry, 53, 111-122.

Silva, B. M., Andrade, P. B., Mendes, G. C., Seabra, R. M., \& Ferreira, M. A. (2002). Study of the organic acids composition of quince (Cydonia oblonga Miller) fruit and jam. Journal of Agricultural and Food Chemistry, 50, 2313-2317.

Silva, B. M., Andrade, P. B., Mendes, G. C., Valentão, P., Seabra, R. M., \& Ferreira, M. A. (2000). Analysis of phenolic compounds in the evaluation of commercial quince jam authenticity. Journal of Agricultural and Food Chemistry, 48, 2853-2857.

Silva, B. M., Andrade, P. B., Seabra, R. M., \& Ferreira, M. A. (2001). Determination of selected phenolic compounds in quince jams by solid-phase extraction and HPLC. Journal of Liquid Chromatography E Related Technologies, 24(18), 2861-2872.

Silva, B. M., Andrade, P. B., Seabra, R. M., Oliveira, M. B. P. P., Ferreira, M. A., \& Ferreres, F. (2005). Composition of quince (Cydonia oblonga Miller) seeds: Phenolics, organic acids and free amino acids. Natural Products Research, 19, 275-281.

Silva, B. M., Andrade, P. B., Valentão, P., Ferreres, F., Seabra, R. M., \& Ferreira, M. A. (2004). Quince (Cydonia oblonga Miller) fruit (pulp, peel, and seed) and jam: Antioxidant activity. Journal of Agricultural and Food Chemistry, 52, 4405-4712.

Silva, B. M., Andrade, P. B., Valentão, P., Mendes, G. C., Seabra, R. M., \& Ferreira, M. A. (2000). Phenolic profile in the evaluation of commercial quince jellies authenticity. Food Chemistry, 71, 281-285.

Silva, B. M., Casal, S., Andrade, P. B., Seabra, R. M., Oliveira, M. B., \& Ferreira, M. A. (2003). Development and evaluation of a GC/FID method for the analysis of free amino acids in quince fruit and jam. Analytical Science, 19, 1285-1290.

Silva, B. M., Valentão, P., Seabra, R. M., Andrade, P.B. (in press). Quince (Cydonia oblonga Miller): An interesting dietary source of bioactive compounds. Food Chemistry Research Developments.

Sousa, C., Silva, B. M., Andrade, P. B., Valentão, P., Silva, A., Ferreres, F., et al. (2007). Homo-monoterpenic compounds as chemical markers for Cydonia oblonga Miller. Food Chemistry, 100, 331-338

Valentão, P., Andrade, P. B., Rangel, J., Ribeiro, B., Silva, B. M., Baptista, P., et al. (2005). Effect of the conservation procedure on the contents of phenolic compounds and organic acids in chanterelle (Cantharellus cibarius) mushroom. Journal of Agricultural and Food Chemistry, 53, 4925-4931.

Valentão, P., Lopes, G., Valente, M., Barbosa, P., Andrade, P. B., Silva, B. M., et al. (2005). Quantification of nine organic acids in wild mushrooms. Journal of Agricultural and Food Chemistry, 53, 3626-3630.

Vrchovská, V., Sousa, C., Valentão, P., Ferreres, F., Pereira, J. A., Seabra, R. M., et al. (2006). Antioxidant properties of tronchuda cabbage (Brassica oleracea L. var. costata DC) external leaves against DPPH, superoxide radical, hydroxyl radical and hypochlorous acid. Food Chemistry, 98, 416-425.

Yildirim, H. T. (2006). Evaluation of colour parameters and antioxidant activities of fruit wines. International Journal of Food Science and Nutrition, 57, 47-63.

Wang, X., Jia, W., Zhao, A., \& e Wang, X. (2006). Anti-influenza agents from plants and traditional Chinese medicine. Phytotherapy Research, 20, 335-341.

Wildman, R. E. C. (2001). Nutraceuticals: A brief review of historical and teleological aspects. In Handbook of nutraceuticals and functional foods. Florida, Boca Raton: CRC Press. 